\title{
Cellular Automata Simulation of Site-saturated and Constant Nucleation Rate Transformations in Three Dimensions
}

\author{
Paulo Rangel Rios*, Valmir Torres de Oliveira, Luciana de Oliveira Pereira, \\ Maxwell Ruela Pereira, José Adilson de Castro \\ Universidade Federal Fluminense, \\ Escola de Engenharia Industrial Metalúrgica de Volta Redonda, \\ Av. dos Trabalhadores, 420, 27255-125 Volta Redonda - RJ, Brazil
}

Received: September 27, 2005; Revised: April 3, 2006

\begin{abstract}
Microstructural evolution in three dimensions of nucleation and growth transformations is simulated by means of cellular automata. Two types of nucleation are considered: site-saturated nucleation and constant nucleation rate. The simulated microstrutural evolution agrees very well with exact analytical expressions. The simulated data also gives very good agreement with expressions derived to describe the evolution of the interfaces between transformed grains.
\end{abstract}

Keywords: microstructure, kinetics, recrystallization, computer simulation, cellular automata

\section{Introduction}

Formal kinetics is a powerful technique to model nucleation and growth solid state transformations. In these transformations, a parent phase, $\alpha$, also called "matrix" or untransformed region", transforms into a product phase, $\beta$, also called "transformed region". In this approach, nucleation and growth are regarded as purely "operational" concepts meaning that no assumption is made regarding their mechanism but only their geometrical and kinematical characteristics. Recrystallization kinetics in particular has been mainly analyzed with the help of formal kinetics methodology ${ }^{1-4}$.

This approach has two facets: one experimental another theoretical. The experimental facet specifies the microstrutural descriptors 5 that may be experimentally obtained, preferentially from planar sections, such as: volume fraction transformed, $V_{V}$; interface area between transformed and untransformed regions, $S_{V}$, and interface area between transformed regions, $S_{V \beta}$. The theoretical facet aims at developing expressions that involve these measurable quantities. Today, one attempts not to rely on three-dimensional quantities that cannot be measured by standard stereological methods but require tedious and time consuming serial sectioning. With the advent of 3DXRD techniques ${ }^{6}$ experimental determination of three-dimensional quantities like the number of nuclei per unit of volume, $N_{V}$, or even nuclei location in space might well become viable on a routine basis.

The basis of formal kinetic modeling is the early work of Johnson$\mathrm{Mehl}^{7}$, Avrami $^{8-10}$ and Kolmogorov ${ }^{11}$ (JMAK), which used only a single microstructural descriptor, $V_{V^{\prime}}$. JMAK's work was subsequently extended by DeHoff ${ }^{12}$, who proposed the use of an additional microstructural descriptor, $S_{V}$, and the associated concept of microstructural path. Vandermeer and coworkers ${ }^{13}$ extended DeHoff's microstructural path concept and crystallized it in an all round theoretical treatment covering variable nucleation and growth rates as well as non-spherical regions.

Nevertheless, even general analytical treatments have significant limitations owing to unavoidable simplifying assumptions. For instance, Vandermeer et al. ${ }^{13}$ assume that nuclei are randomly located in space. However, when one considers, for example, austenite to ferrite transformation, ferrite nucleates at grain boundaries ${ }^{14,15}$. Another example is recrystallization, where the deformed state is, as a rule, highly heterogeneous ${ }^{16}$ casting some doubt on the assumption that nuclei are randomly located in space. Relaxing this and other assumptions may lead to situations too complex for analytical methods. In this regard, computer simulation of microstructural evolution can be an invaluable tool since it can simulate situations beyond analytical treatment. Compared to other simulation methods, cellular automata ${ }^{17-30}$ is a convenient choice for phase transformations in general and recrystallization in particular ${ }^{23}$.

Computer simulation aims at describing transformation in complex situations for which there is no analytical theory. In order to accomplish this, one must first make sure that the simulation is able to describe accurately the simplest cases for which there are exact mathematical descriptions. Interestingly, this basic or minimum validation of the simulation has been rarely reported in published papers ${ }^{23}$.

In previous papers ${ }^{28,29}$, present authors carried out cellular automata (CA) simulation of phase transformation/recrystallization in two dimensions (2D). Earliest computer simulations of microstructural evolution were usually performed in two dimensions. Even in recent papers some authors have chosen to work in two dimensions probably because the computer cost of three-dimensional (3D) simulations is significantly higher than that of $2 \mathrm{D}$ simulations. Although there is a certain interest in the study of two-dimensional transformations, for example, transformations taking place in thin films or thin sheets, the greatest interest lye in three dimensional transformations.

The purpose of this paper is to simulate phase transformations/ recrystallization in $3 \mathrm{D}$ by cellular automata (CA). Two basic nucleation types were assumed:

a) site-saturation: all nucleation occurs at the beginning of the transformation; and

b) constant nucleation rate: the number of new nuclei per unit of time per unit of volume is constant.

Simulated microstrutural evolution was compared in detail with predictions of mathematically exact analytical theories ${ }^{28}$. Moreover, simulated data was used to test new expressions recently derived by Rios et al..$^{31}$ to describe the evolution of the interfaces between transformed grains as a function of time or volume fraction transformed. 


\section{Description of the Cellular Automata Simulation}

Cellular automata simulation used a 3D von Neumann neighborhood ${ }^{17}$. The matrix consisted of a cubic lattice with $304 \times$ $304 \times 304$ cells. One cell edge was considered to have a unit length and consequently of a single cell had unit volume. Units of all quantities reported here follow from this. The nuclei were randomly dispersed on the matrix. For site-saturated nucleation, the number of nuclei per unit of volume, $N_{V}$, was equal to $125 / 304^{3}$. For constant nucleation rate, the nucleation rate per unit of time per unit of volume, $I$, was equal to $2 / 304^{3}$. This nucleation rate resulted in 141 nuclei per unit of volume when the transformation reached $V_{V}=1$.

The simulation produced a sequence of matrices as a function of time. Time is discrete in CA, it takes integer values starting from $t=0$. One time unit corresponds to the interval between two consecutive matrix updates ${ }^{17}$. From the simulated matrices, all the desired quantities could be extracted. Oliveira ${ }^{30}$ gives further details of the present simulation.

\section{Growth of a Single Grain}

When a single grain grows in isolation, it is possible to find analytical expressions for its growth. The following expressions are specific for the $3 \mathrm{D}$ von Neumann neighborhood adopted in this simulation. The time origin, $t=0$, corresponds to a volume fraction equal to zero, $V_{V}=0$. Therefore, the volume of a single grain, $v$, and its area, $s$, at $t=0$ are equal to:

$$
\begin{aligned}
& v(0)=0 \\
& s(0)=0
\end{aligned}
$$

For $t \geq 1$ the volume of a single grain as a function of time is:

$v(t)=\frac{4}{3} t^{3}-2 t^{2}+\frac{8}{3} t-1$

This equation is not valid for $t=0$. Neglecting the lower order powers of time:

$$
v(t) \cong \frac{4}{3} t^{3} \text { units of volume }
$$

The area of a single grain, $s$, for $t \geq 1$ is:

$s(t)=12 t^{2}-12 t+6$

Again, neglecting early time behavior:

$s(t) \cong 12 t^{2}$ area units

Equations 3 and 5 will be used below in section 4 to represent the growth of a single grain.

The average interface velocity, $\langle G\rangle$, can be calculated with the help of Cahn-Hagel ${ }^{32}$ equation:

$<G>=\frac{\iint G d s}{\iint d s}=\frac{1}{s} \frac{d v}{d t}$

Inserting Equations 3 and 5 gives:

$<G>\cong \frac{1}{3}$ units of length/unit time

The units will be omitted from now on.

The average interface velocity is constant. Therefore, for a single grain the simulation was able to produce constant growth rate.

It is worthy mentioning that the volume and the area of a single grain showed time dependencies different from what would be expected from constant velocity growth. In this case it was possible to use only the leading term, with the highest power of time. A similar situation was found in a previous work ${ }^{28}$ on $2 \mathrm{D}$ cellular automata simulation. It is not easy to say what effect a different choice of neighborhood or a different transition criterion would have on this time dependence. So, CA simulations should check this. This is rarely reported in published papers on this subject ${ }^{17-30}$.

\section{Microstructural Evolution: Volume Fraction, Interface Area Per Unit of Volume and Microstructural Path}

In this section, the expressions derived in the previous section for single grain evolution were used to obtain expressions containing "global" measurements, that is, average measurements such as volume fraction and interface area per unit of volume. The calculated expressions were compared with the results from the simulation. This section mainly deals with quantities related to the mobile interface: the interface between transformed and untransformed regions. In each case, an analytical expression was derived for site-saturation nucleation and constant nucleation rate. Section 5 will compare simulations results with new expressions derived for the evolution of the interfaces between transformed regions.

Figures 1-3 show the results obtained from the simulation compared with the theoretical expressions obtained in this section. One

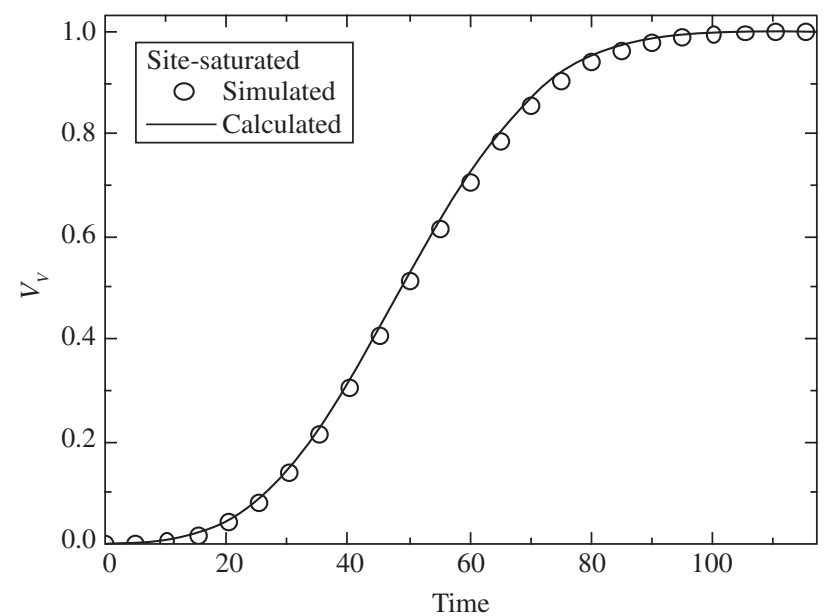

(a)

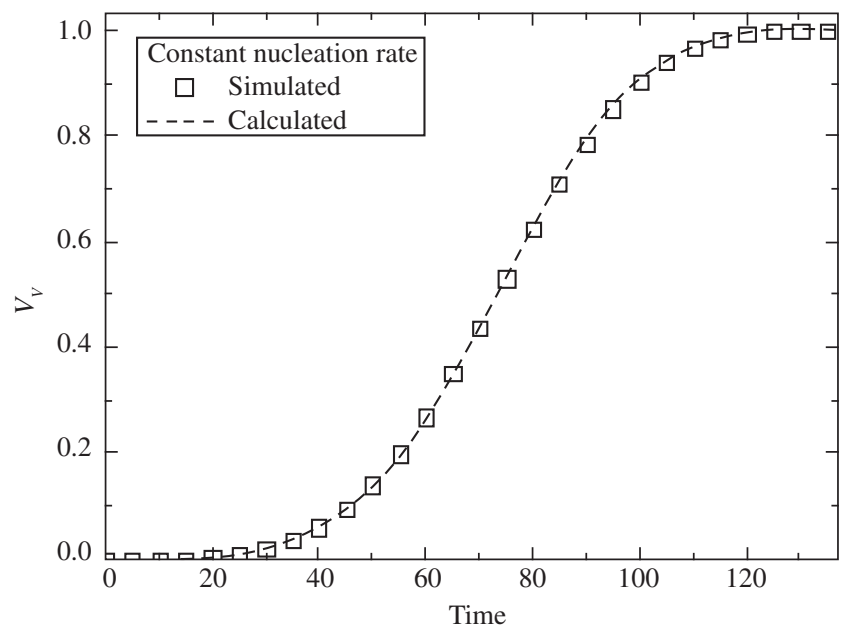

(b)

Figure 1. Volume fraction, $V_{v}$, against time. There is good agreement between theory and simulation: a) site-saturated; and b) constant nucleation rate. 
cannot directly compare Figures $1 \mathrm{a}-3 \mathrm{a}$ with Figures $1 \mathrm{~b}-3 \mathrm{~b}$ because the number of nuclei per unit of volume is different in each case. In order to avoid overloading the figures not all simulation points were plotted.

\subsection{Volume fraction as a function of time}

The extended volume fraction, $V_{V E}$, is the sum of the volume of all individual grains supposing that they grow without impingement. The subscript " $E$ " is reserved for "extended quantities" defined in a manner similar to the extended volume fraction. Using Equation 3 one obtains:

$$
\begin{aligned}
& V_{V E}=\frac{4}{3} N_{V} t^{3} \\
& V_{V E}=\frac{1}{3} I t^{4}
\end{aligned}
$$

where $N_{V}$ is the number of nuclei per unit of volume and $I$ is the nucleation rate per unit of volume; Equations $8 \mathrm{a}$ and $8 \mathrm{~b}$ correspond to site-saturated nucleation and constant nucleation rate, respectively.

For randomly located nuclei, the extended volume fraction, $V_{V E}$, and the real volume fraction, $V_{V}$, are related by the JMAK expression:

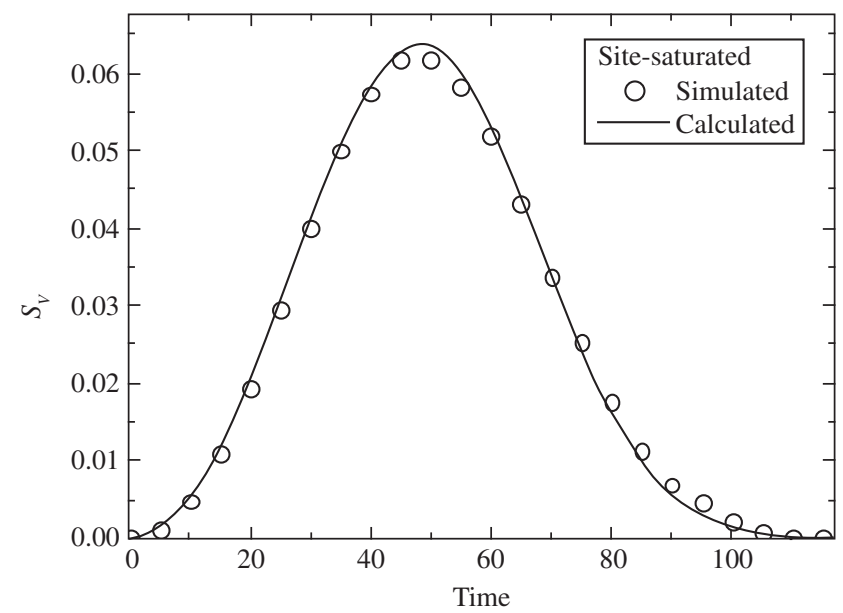

(a)
$V_{V}=1-\exp \left(-V_{V E}\right)$

As a result:

$$
\begin{aligned}
& V_{V}=1-\exp \left(-\frac{4}{3} N_{V} t^{3}\right) \\
& V_{V}=1-\exp \left(-\frac{1}{3} I t^{4}\right)
\end{aligned}
$$

where Equations 10a and 10b correspond to site-saturated nucleation and constant nucleation rate, respectively.

Figures $1 \mathrm{a}$ and $1 \mathrm{~b}$ show a comparison between $V_{V}$ calculated from the analytical solution and obtained from the CA simulation. The agreement is very good.

\subsection{Interface area between transformed and untransformed regions as a function of time and volume fraction}

The extended interface area per unit of volume between transformed grains and untransformed regions, $S_{V E}$, is the sum of the interface area of all individual grains supposing that they grow without impingement. Using Equation 5 it can beshown that:

$$
S_{V E}=12 N_{V} t^{2}
$$

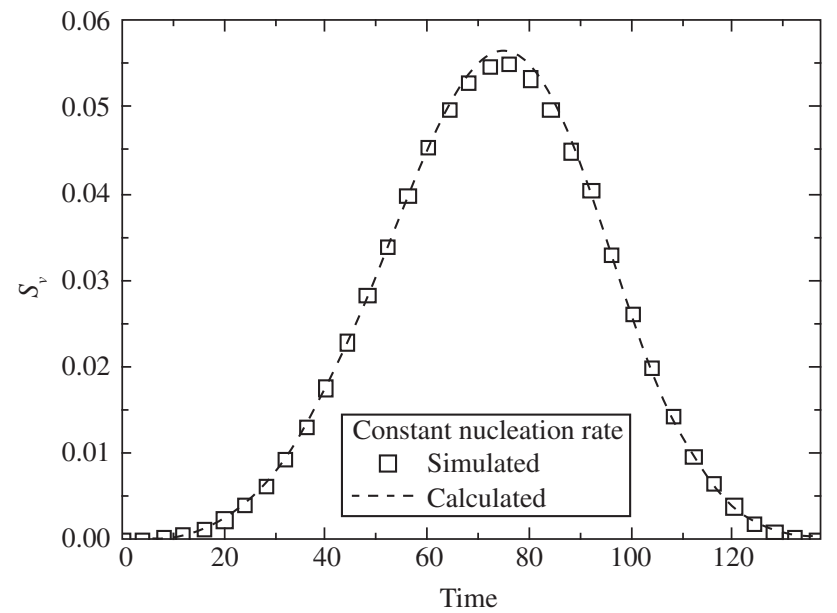

(b)

Figure 2. Interface area between transformed and untransformed region per unit of volume, $S_{V}$, against time. There is good agreement between theory and simulation: a) site-saturated; and b) constant nucleation rate.

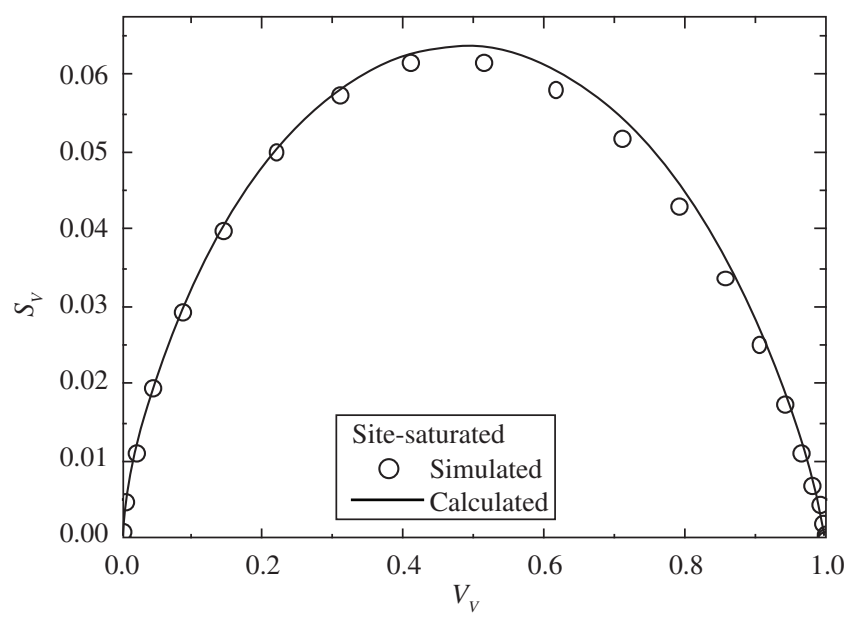

(a)

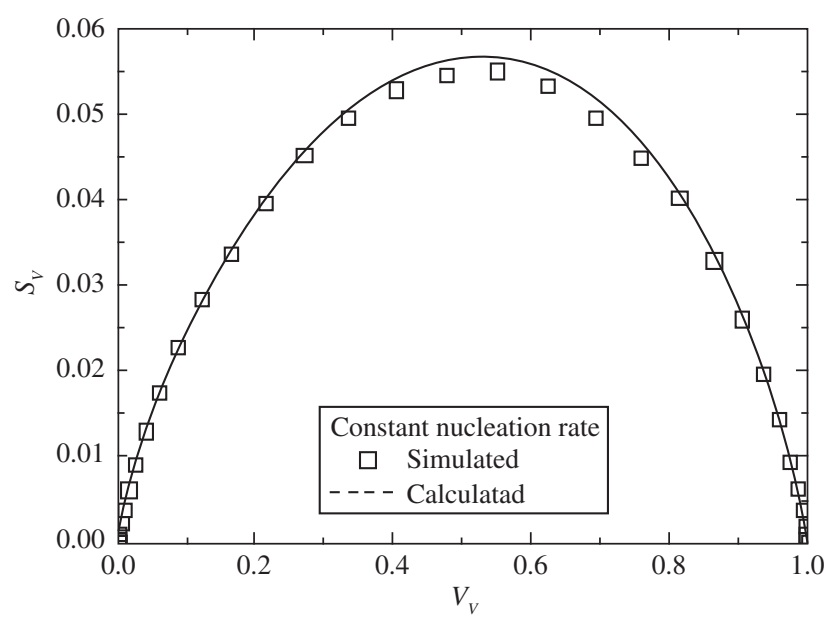

(b)

Figure 3. Microstructural path: area of the interface between transformed and untransformed region per unit of volume, $S_{V}$, against volume fraction, $V_{V}$. There is good agreement between theory and simulation: a) site-saturated; and b) constant nucleation rate. 


$$
S_{V E}=4 I t^{3}
$$

where Equations 11a and $11 \mathrm{~b}$ correspond to site-saturated nucleation and constant nucleation rate, respectively.

The extended interface area and real interface area, $S_{V}$, are related by the DeHoff's ${ }^{12}$ expression:

$$
S_{V}=\left(1-V_{V}\right) S_{V E}
$$

Equation 12 together with Equations 1a and 1b give:

$$
\begin{aligned}
& S_{V}=12 N_{V} t^{2} \exp \left(-\frac{4}{3} N_{V} t^{3}\right) \\
& S_{V}=4 I t^{3} \exp \left(-\frac{1}{3} I t^{4}\right)
\end{aligned}
$$

where Equation 13a and 13b correspond to site-saturated nucleation and constant nucleation rate, respectively.

Figures $2 \mathrm{a}$ and $2 \mathrm{~b}$ show a comparison between the $S_{V}$, calculated from the analytical solution, Equations 13a-b, and obtained from the CA simulation. The agreement is very good.

In three dimensions the microstructural path can be seen as a curve on the $\left(V_{V}, S_{V}\right)$ plane. From Equations 8a,b and 11a,b one can find a relationship between $S_{V E}$ and $V_{V E}$ :

$$
\begin{aligned}
& S_{V E}=3\left(36 N_{V}\right)^{\frac{1}{3}} V_{V E}^{\frac{2}{3}} \\
& S_{V E}=4(27 I)^{\frac{1}{4}} V_{V E}^{\frac{3}{4}}
\end{aligned}
$$

where Equation 14a and 14b correspond to site-saturated nucleation and constant nucleation rate, respectively. From Equations 9, 12 and Equations 14 one can obtain $S_{V}$ as a function of volume fraction transformed:

$$
\begin{aligned}
& S_{V}=3\left(36 N_{V}\right)^{\frac{1}{3}}\left(1-V_{V}\right)\left[\ln \left(\frac{1}{1-V_{V}}\right)\right]^{\frac{2}{3}} \\
& S_{V}=4\left(27 I_{0}\right)^{\frac{1}{4}}\left(1-V_{V}\right)\left[\ln \left(\frac{1}{1-V_{V}}\right)\right]^{\frac{3}{4}}
\end{aligned}
$$

where Equation $15 \mathrm{a}$ and $15 \mathrm{~b}$ correspond to site-saturated nucleation and constant nucleation rate, respectively.

Figures $3 \mathrm{a}$ and $3 \mathrm{~b}$ show a comparison between the values calculated using the theoretical expression for the microstructural path obtained above and those obtained from the simulation. The agreement is very good.

The average grain boundary velocity is given by ${ }^{32}$ :

$$
<G>=\frac{1}{s} \frac{d v}{d t}=\frac{1}{S_{V E}} \frac{d V_{V E}}{d t}=\frac{1}{S_{V}} \frac{d V_{V}}{d t}=\frac{1}{3}
$$

The grain boundary velocity calculated from the simulated values was $0.34+0.01$, very close to the theoretical prediction for both sitesaturated nucleation and constant nucleation rate. This means that the interface velocity in real space is the same as that in extended space, as expected.

In summary CA simulation showed very good agreement with exact mathematical expressions.

\section{Comparison of the Simulation with New Analytical Expressions for the Impinged Interface Area Per Unit of Volume, Total Interface Area Per Unit of Volume and the Contiguity}

There are two kinds of interface during an heterogeneous phase transformation: mobile and immobile. The mobile interface is the interface between the transformed region and the untransformed matrix. This interface migrates and its migration determines the transformation progress. The immobile interface is a consequence of impingement of transformed grains. The transformed grain size is a function of the interface area between transformed grains when the transformation reaches $V_{V}=1$.

Traditionally, experimental and theoretical studies focus on the mobile interface. This could be seen in the previous section where all equations involved only $S_{V}$. However, the amount of interface between transformed grains, $S_{V \beta}$, is an important quantity and contains valuable information on transformation evolution. CA simulation is able to provide accurate data on these interfaces and so gives a good basis for comparison with analytical models. Combining $S_{V \beta}$ with $S_{V}$ two useful quantities may be defined. One is the total interface area per unit of volume, $S_{V T}$ :

$$
S_{V T}=S_{V}+2 S_{V \beta}
$$

Another important quantity, derived from $S_{V \beta}$ with $S_{V}$ is the contiguity parameter, $C_{\beta}$. The contiguity is the ratio of immobile interface area to the total interface area and is quite sensitive to deviations of nuclei location from randomness, as recently shown by Vandermeer and Jensen ${ }^{5,33}$; see also Rios et al. ${ }^{29}$. It can be defined as ${ }^{5}$ :

$$
C_{\beta}=\frac{2 S_{V \beta}}{S_{V}+2 S_{\nu \beta}}
$$

Recently Rios et al. ${ }^{31}$ have derived analytical expressions for $S_{V \beta}$ as a function of volume fraction transformed for time dependent nucleation rate and time dependent velocity. They followed up the earlier work by Meijring ${ }^{34}$, Gokhale ${ }^{35,36}$ and more recently an elegant mathematical derivation by Tong et al. ${ }^{37}$. All these authors solved this problem for spherical growth. Rios et al. ${ }^{31}$ derivation has the advantage of being mathematically simpler and not restricted to spherical growing regions. The disadvantage of their method is that, as will be shown below, they do not obtain an explicit expression for $S_{V \beta F}$, the value of $S_{V \beta}$ for a fully transformed matrix. Their expressions can be used to obtain analytical expressions for $S_{V T}$ and $C_{\beta}$. Rios et al. ${ }^{31}$ derivation is repeated here for convenience. The starting point of their derivation was DeHoff's relationship between the extended and the real interface area, Equation 12, rewritten below in a slightly different way:

$$
S_{V}\left(V_{V}, S_{V E}\right)=S_{V E}\left(1-V_{V}\right)
$$

One can consider that a small change in $S_{V}, \delta S_{V}$, can be seen as a sum of changes in each variable, $\delta V_{V}$ and $\delta S_{V E}$ :

$$
\delta S_{V}\left(\frac{\partial S_{V}}{\partial S_{V E}}\right)_{V_{V}} \delta S_{V E}+\left(\frac{\partial S_{V}}{\partial V_{V}}\right)_{S_{V E}} \delta V_{V}
$$

Or

$\delta S_{V}=\left(1-V_{V}\right) \delta S_{V E}-\left(S_{V E}\right) \delta V_{V}$

The above has two terms:

a) The first is always positive, it corresponds to the increase in $S_{V}$ when the interface length increases $\delta S_{E}$ in extended space, at a constant $V_{V}$, corrected for impingement by the factor $\left(1-V_{V}\right) ;$ and

b) The second term, $-S_{V E} \delta V_{V}$, is negative because $S_{V E}$ and $\delta V_{V}$ are always positive. It corresponds to the decrease in $S_{V}$, when the area fraction increases for a constant $S_{V E}$, this decrease corresponds to a "loss" in $S_{V}$ due to the "creation" of new impinged interface. Two extended interfaces meet to make one impinged interface, $S_{V \beta}$, so:

$$
\frac{d S_{V \beta}}{d V_{V}}=\alpha \frac{S_{E}}{2}
$$

where $\alpha$ is a normalizing factor to be determined below.

Integrating Equation 22 gives: 


$$
S_{V \beta}=\frac{\alpha}{2} \int_{0}^{V_{V}} S_{V E} d V_{V}
$$

It is more convenient to write Equation 23 in terms of extended quantities with the help of a relationship obtained from Equation 9:

$$
d V_{V}=\exp \left(-V_{V E}\right) d V_{V E}
$$

where $V_{V E}$, the extended volume fraction, is the sum of the volume of all growing grains divided by the total volume assuming that the growing grains do not impinge. Equation 23 then becomes:

$$
S_{V \beta}=\frac{\alpha}{2} \int_{0}^{V_{E}} S_{V E} \exp \left(-V_{V E}\right) d V_{V E}
$$

In order to integrate Equation 25 it is necessary to know the relationship between $S_{V E}$ and $V_{V E}$ which is supposed to be of the form ${ }^{13}$ :

$S_{V E}=C\left(V_{V E}\right)^{q}$

Resulting in:

$$
S_{V \beta}=\frac{\alpha C}{2} \int_{0}^{V_{V E}}\left(V_{V E}\right)^{q} \exp \left(-V_{V E}\right) d V_{V E}
$$

This integral can be written in terms of the incomplete Gamma function, $\Gamma$ :

$$
S_{V \beta}=\frac{\alpha C}{2}\left(\Gamma(q+1)-\Gamma\left(q+1, V_{V E}\right)\right)
$$

The factor $\alpha$ can be found by taking the limit $V_{V E} \rightarrow \infty$. For $V_{V E} \rightarrow \infty, V_{V} \rightarrow 1$, the specimen is fully transformed and $S_{V \beta}$ tends to a constant value $S_{V \beta F}$ :

$$
\alpha=\frac{2 S_{V \beta F}}{C \Gamma(a+1)}
$$

Inserting Equation 29 into 26:

$$
\begin{aligned}
& \frac{S_{V \beta}}{S_{V \beta F}}=\left(1-\frac{\Gamma\left(q+1, V_{V E}\right.}{\Gamma(q+1)}\right)= \\
& \left(1-\frac{\Gamma\left(q+1,-\ln \left(1-V_{V}\right)\right)}{\Gamma(q+1)}\right)
\end{aligned}
$$

where $S_{V \beta F}$ is the amount of interface area between transformed regions when the transformation has reached $V_{V}=1$. Using the definition of contiguity, Equation 18:

$$
C_{\beta}=\frac{2 S_{V \beta F}\left(1-\frac{\Gamma\left(q+1, \ln \left(\frac{1}{1-V_{V}}\right)\right)}{\Gamma(q+1)}\right)}{C\left(1-V_{V}\right)\left(\ln \left(\frac{1}{1-V_{V}}\right)\right)^{q}+2 S_{V \beta F}\left(1-\frac{\Gamma\left(q+1, \ln \left(\frac{1}{1-V_{V}}\right)\right)}{\Gamma(q+1)}\right)}
$$

For the cases considered here, $C$ and $q$ can be found from the relationships between $S_{V E}$ and $V_{V E}$ given by Equations 14a-b:

a) site-saturation: $q=2 / 3$ and $C=3\left(36 N_{V}\right)^{1 / 3}$; and

b) constant nucleation rate: $q=3 / 4$ and $C=4(27 I)^{1 / 4}$. al. ${ }^{31}$.

More general expressions for $C$ and $q$ can be found in Rios et

Equations 30 and 31 can be written as a function of time with the help of Equations 10a and b. The total interface area per unit of volume, $S_{V T}$, can be found by means of Equations 15a,b and Equations 30 and Equation 17.

Figures 4-7 show the comparison of the values calculated by means of Equations 17, 30 and 31 and those obtained from the simulation. The agreement is very good. For $S_{V \beta}$ and $S_{V T}$, Figures 4-7, the normalized quantities $S_{V \beta} / S_{V \beta F}$ and $S_{V I} /\left(2 S_{V \beta F}\right)$ were plotted. In order to avoid overloading the figures not all simulation points were plotted.
An interesting aspect of the present comparison is that the growing regions were not spherical in this simulation. This shows that Rios et al. ${ }^{31}$ expression applies when the shape of the growing regions is not spherical $^{34-36}$.

Figures $5 \mathrm{c}$ and $7 \mathrm{c}$ show that the normalized quantities $S_{V \beta} / S_{V B F}$ and $C_{\beta}$ are practically the same for both nucleation types. Randomness in nuclei distribution might have an underlying effect that is stronger than the details of the kinetics ${ }^{31}$.

It is important to mention that no adjustable parameters were used in this work. Only exact analytical solutions were compared with the simulations. The good agreement between theory and simulation shows that CA is able to reproduce situations for which there are mathematically exact solutions. This good agreement suggests that CA may be applied to complex situations for which there are no analytical solutions.

\section{Summary and Conclusions}

First it is worthy mentioning that no adjustable parameters were used throughout this work. Moreover, this work was motivated by recrystallization studies and it is perhaps more useful in that context.

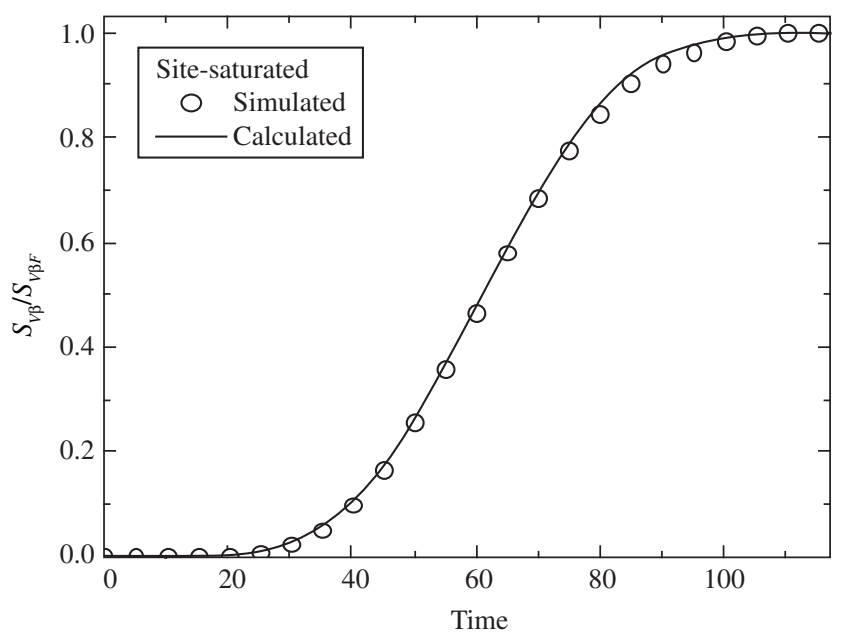

(a)

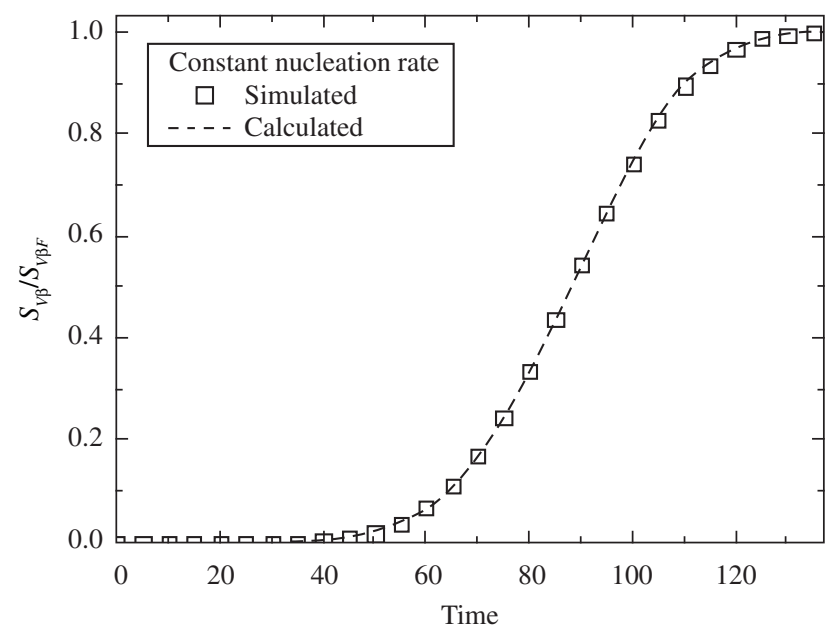

(b)

Figure 4. Area of the interface between transformed and transformed region per unit of volume, $S_{V \beta} / S_{V \beta F}$, against time. There is good agreement between theory and simulation: a) site-saturated; and b) constant nucleation rate. 


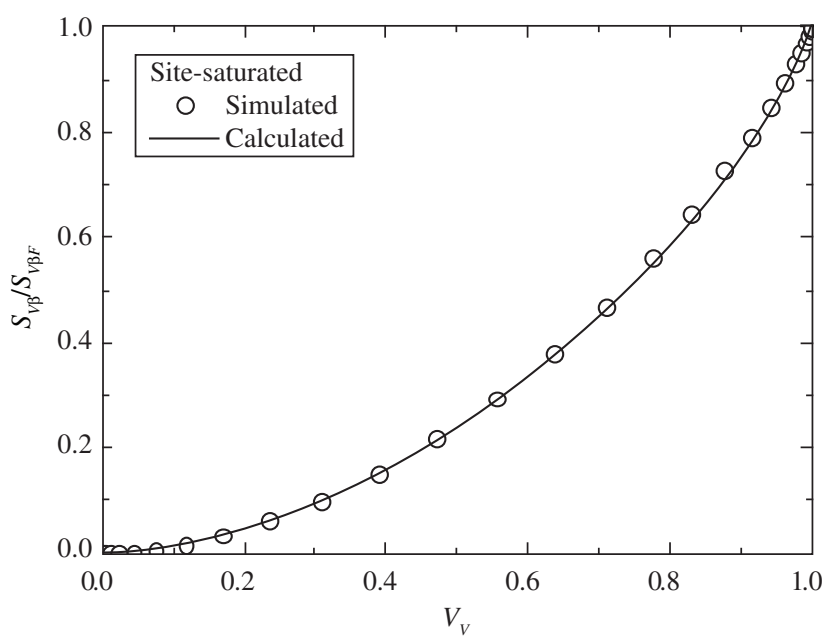

(a)

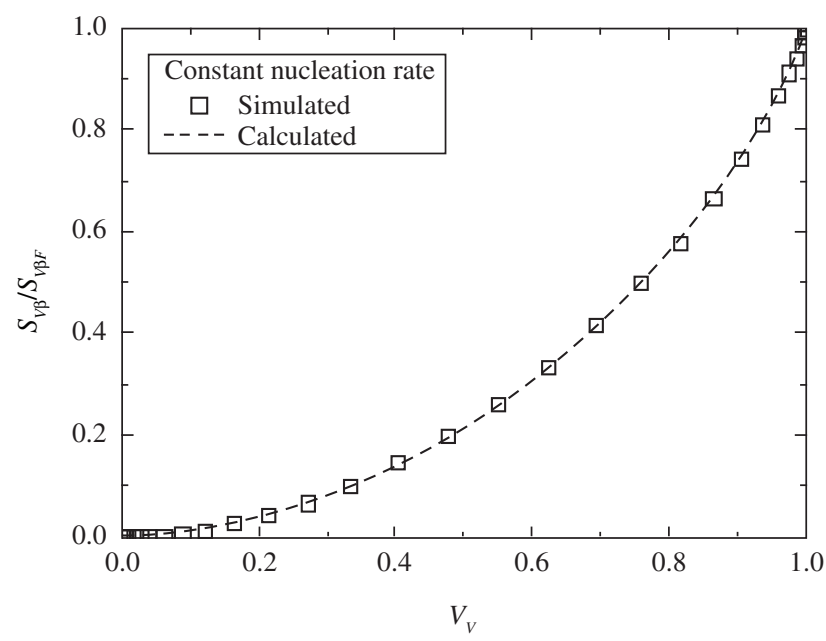

(b)

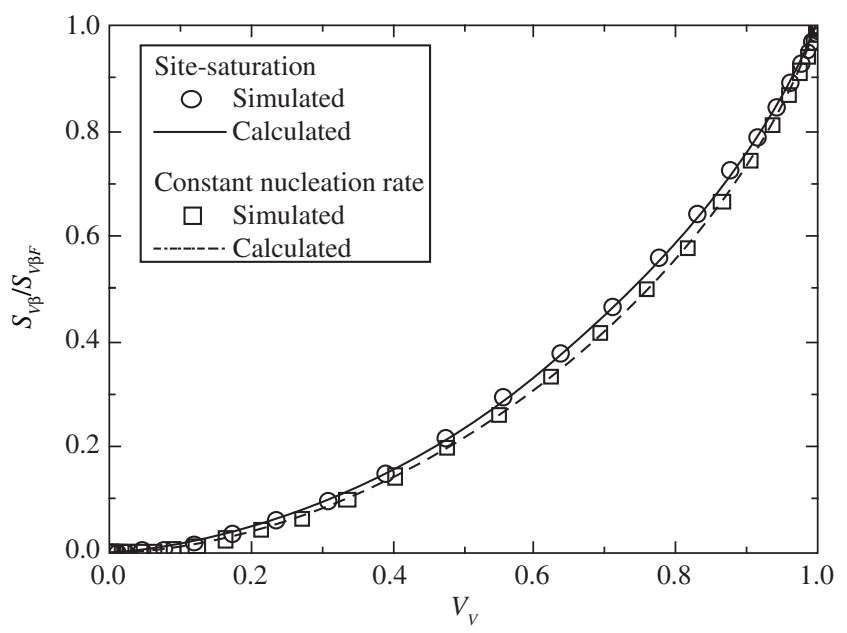

(c)

Figure 5. Area of the interface between transformed and transformed region per unit of volume, $S_{V \beta} / S_{V B F}$, against volume fraction. There is good agreement between theory and simulation: a) site-saturated; b) constant nucleation rate; and c) Figures $5 \mathrm{a}$ and $5 \mathrm{~b}$ plotted together; $S_{V \beta} / S_{V B F}$ as a function of volume fraction is practically the same for both nucleation types.

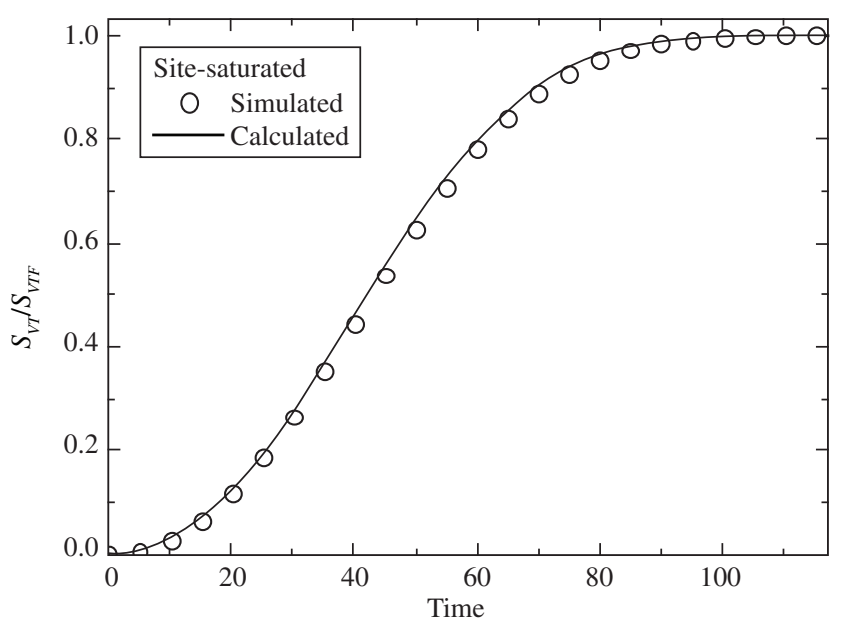

(a)

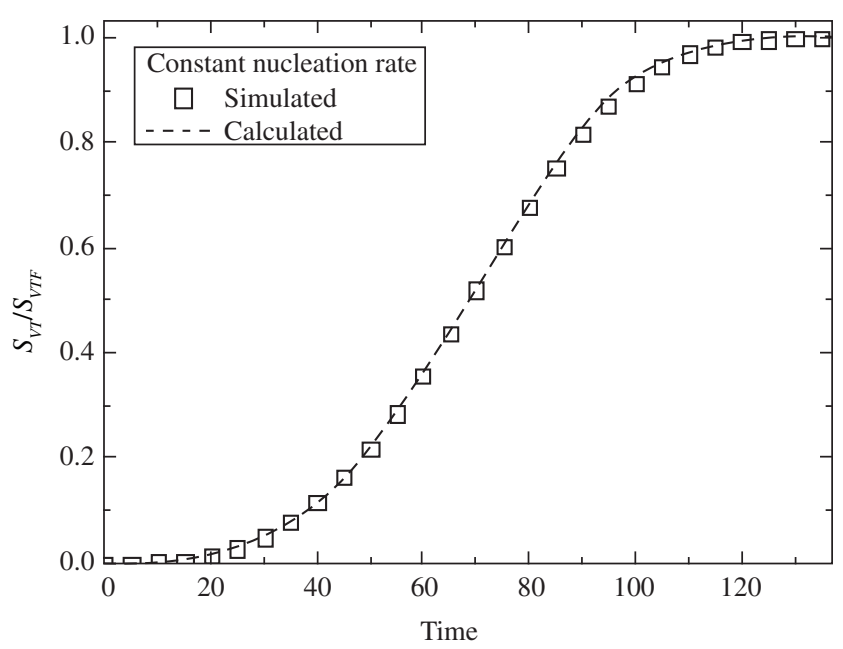

(b)

Figure 6. Total interface area per unit of volume, $S_{V T} /\left(2 S_{V \beta F}\right)$ against time. There is good agreement between theory and simulation: a) site-saturated; and $b)$ constant nucleation rate.

Nonetheless, its results are general and may be applied to any nucleation and growth transformation.

Present results show that CA is able to reproduce the site-saturated nucleation and constant nucleation rate kinetics for which there are exact solutions. This good agreement suggests that CA may be used with some confidence in more complex situations for which there are no theoretical results.

CA simulation showed to be useful to test expressions developed by Rios et al. ${ }^{31}$ for the evolution of area per unit of volume of the interface between the transformed regions. The good agreement observed is a reconfirmation of the validity of these new expressions in the situations examined here.

\section{Acknowledgments}

This work was supported by Conselho Nacional de Desenvolvimento Científico e Tecnológico, CNPq, Coordenação de Aperfeiçoamento de Pessoal de Nível Superior, CAPES, and Fundação de Amparo à Pesquisa do Estado do Rio de Janeiro, FAPERJ. 


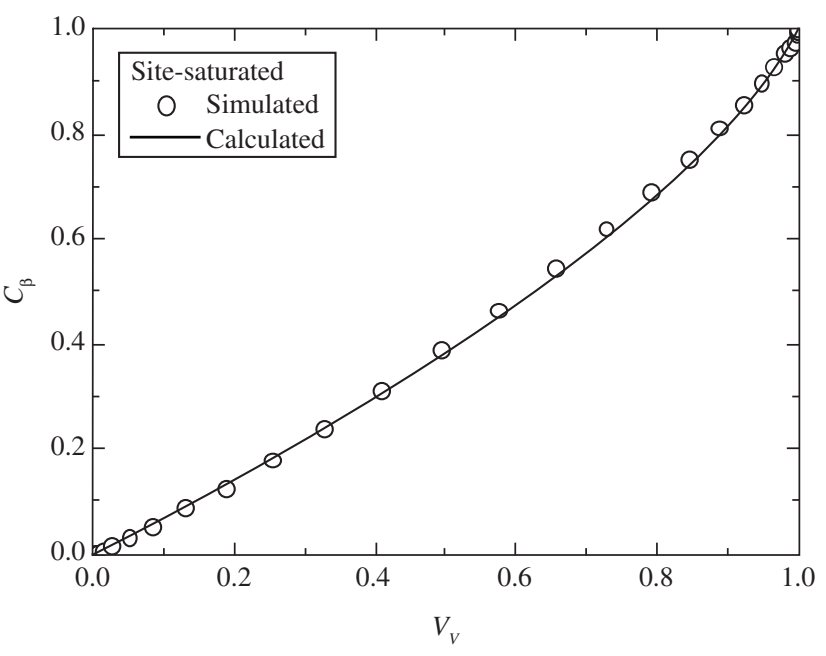

(a)

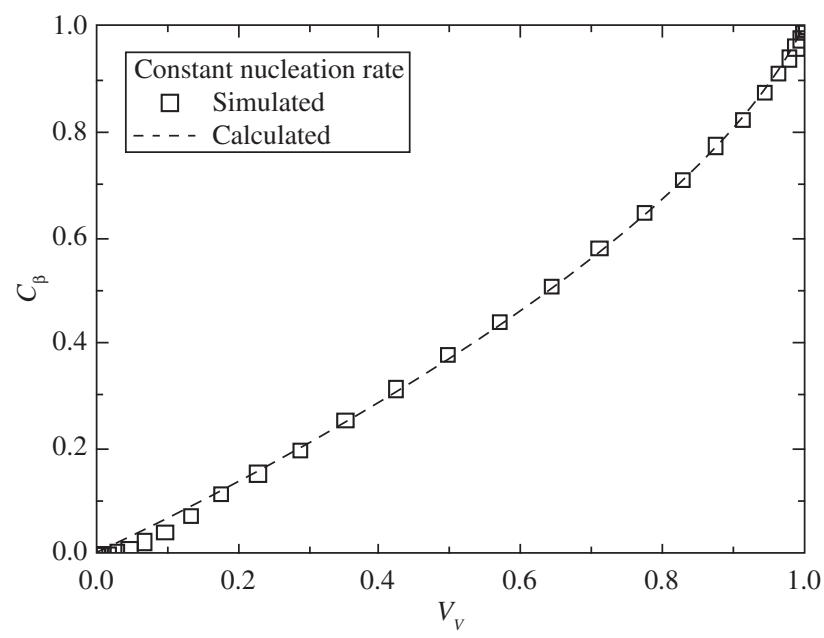

(b)

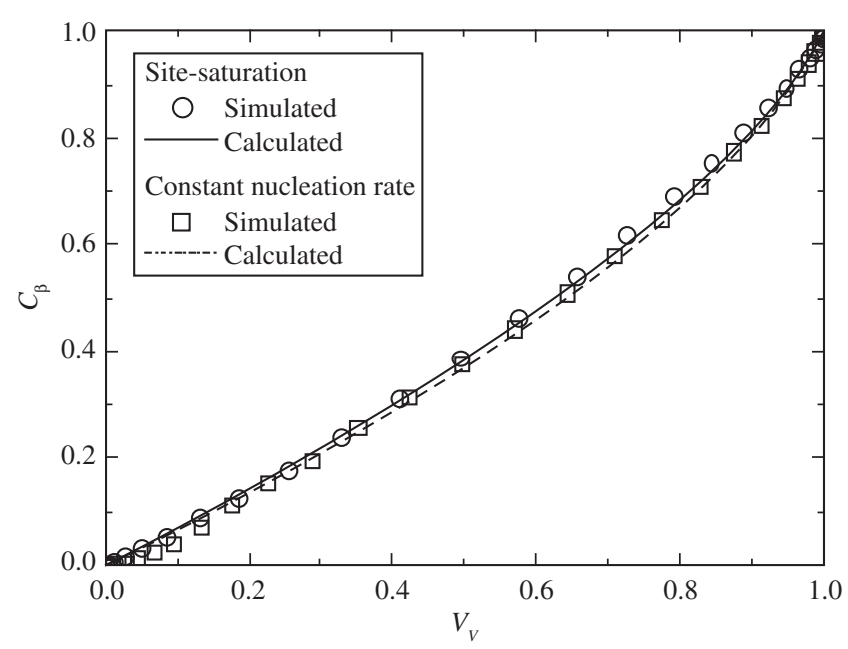

(c)

Figure 7. Contiguity, $C_{\beta}$, against volume fraction. There is good agreement between theory and simulation: a) site-saturated nucleation; b) constant nucleation rate; and c) Figures $7 \mathrm{a}$ and $7 \mathrm{~b}$ plotted together; $C_{\beta}$ as a function of volume fraction is practically the same for both nucleation types.

\section{References}

1. Vandermeer RA, Jensen DJ. Microstructural path and temperature dependence of recrystallization in commercial aluminum. Acta Materialia. 2001; 49(11):2083-2094.

2. Rios PR, Padilha AF. Microstructural path of recrystallization in a commercial Al-Mn-Fe-Si (AA3003) alloy. Materials Research. 2003; 6(4):605-613.

3. Rios PR, Padilha AF. Comment on "Microstructural path and temperature dependence of recrystallization in commercial aluminum". Scripta Materialia. 2003; 48(11):1561-1564.

4. Rios PR, Siciliano Jr F, Sandim HRZ, Plaut RL, Padilha AF. Nucleation and growth during recrystallization. Materials Research. 2005; 8(3)225238.

5. Vandermeer RA. Microstructural descriptors and the effects of nuclei clustering on recrystallization path kinetics. Acta Materialia. 2005; 53(5):1449-1457.

6. Lauridsen EM, Poulsen HF, Nielsen SF, Jensen DJ. Recrystallization kinetics of individual bulk grains in $90 \%$ cold-rolled aluminum. Acta Materialia. 2003; 51(15):4423-4435.

7. Johnson WA, Mehl RF. Reaction kinetics in processes of nucleation and growth. Transactions AIME. 1939; 135:416-441.

8. Avrami MJ. Kinetics of phase change I general theory. The Journal of Chemical Physics. 1939; 7(12):1103-1112.

9. Avrami MJ. Kinetics of phase change II transformation-time relations for random distribution of nuclei. The Journal of Chemical Physics. 1940; 8(2):214-224.

10. Avrami MJ. Kinetics of phase change III granulation, phase change, and microstructure kinetics of phase change. The Journal of Chemical Physics. 1941; 9(2):177-184.

11. Kolmogorov NA. The statistics of crystal growth in metals. Isvestiia Academii Nauk SSSR - Seriia Matematicheskaia. 1937; 1:333-359.

12. DeHoff RT. In: Hansen N, Jensen DJ, Leffers T, Ralph B, editors. Annealing Processes-Recovery, Recrystallization and Grain Growth. Proceedings of the $7^{\text {th }}$ Conference of Ris $\phi$ National Laboratory; 1986, Roskilde: Denmark. Roskilde: Ris $\varnothing$ National Laboratory; 1986. p. 35-52.

13. Vandermeer RA, Masumura RA, Rath B. Microstructural paths of shapepreserved nucleation and growth transformations. Acta Metallurgica et Materialia. 1991; 39(3):383-389.

14. Cahn JW. The kinetics of grain boundary nucleated reactions. Acta Metallurgica. 1956; 4(9):449-459.

15. Vandermeer RA, Masumura RA. The microstructural path of grain boundary nucleated phase transformations. Acta Metallurgica et Materialia. 1992; 40(4):877-886.

16. Sandim HRZ, Martins JP, Padilha AF. Orientation effects during grain subdivision and subsequent annealing in coarse-grained tantalum. Scripta Materialia. 2001; 45(6):733-738.

17. Hesselbarth HW, Göbel IR. Simulation of recrystallization by cellular automata. Acta Metallurgica et Materialia. 1991; 39(9):2135-2143.

18. Pezzee CF, Dunand DC. The impingement effect of an inert, immobile $2^{\text {nd }}$ phase on the recrystallization of a matrix. Acta Metallurgica et Materialia. 1994; 42(5):1509-1524.

19. Sessa V, Fanfoni M, Tomellini M. Validity of Avrami's kinetics for random and nonrandom distributions of germs. Physical Review B. 1996; 54(2):836-841.

20. Goetz RL, Seetharaman V. Static recrystallization kinetics with homogeneous and heterogeneous nucleation using a cellular automata model. Metallurgical and Materials Transactions A-Physical Metallurgy and Materials Science. 1998; 29(9):2307-2321.

21. Marx V, Reher FR, Gottstein G. Simulation of primary recrystallization using a modified three-dimensional cellular automaton. Acta Materialia. 1999; 47(4):1219-1230.

22. Pineda E, Pradell T, Crespo D. Non-random nucleation and the Avrami kinetics. Philosophical Magazine A-Physics of Condensed Matter Structure Defects and Mechanical Properties. 2002; 82(1):107-121. 
23. Raabe D. Cellular automata in materials science with particular reference to recrystallization simulation. Annual Review Materials Research. 2002; 32:53-76

24. Zhang L, Zhang CB, Wang YM, Wang SQ, Ye HQ A cellular automaton investigation of the transformation from austenite to ferrite during continuous cooling. Acta Materialia. 2003; 51(18):5519-5527.

25. Goetz RL. Particle stimulated nucleation during dynamic recrystallization using a cellular automata model. Scripta Materialia. 2005; 52(9):851856.

26. Rios PR, Carvalho JJS, Salazar TC, Paula FVL, Castro JA. Cellular automata simulation of the effect of nuclei distribution on the recrystallization kinetics. Materials Science Forum. 2004; 467-470: 659-664.

27. Oliveira JCPT. Simulação do efeito da distribuição dos núcleos na cinética e no caminho microestrutural da recristalização pelo método do autômato celular [unpublished M. Sc. thesis]. Volta Redonda: Universidade Federal Fluminense; 2004.

28. Rios PR, Oliveira JCPT, Oliveira VT, Castro JA. Comparison of analytical models with cellular automata simulation of recrystallization in two dimensions. Materials Research. 2005; 8(3):341-345.

29. Rios PR, Oliveira JCPT, Oliveira VT, Castro JA. Microstructural descriptors and cellular automata simulation of the effects of non-random nuclei location on recrystallization in two dimensions. Materials Research. 2006; 9(2).

30. Oliveira VT. Desenvolvimento do Código Computacional para a Simulação da Recrystallização em 3D pelo Método do Autômato Celular. [unpublished M. Sc. thesis]. Volta Redonda: Universidade Federal Fluminense; 2005 .

31. Rios PR, Godiksen R, Schmidt S, Jensen DJ, Vandermeer RA. Analytical expression for the evolution of interfacial area density between transformed grains during nucleation and growth transformation. Scripta Materialia. 2006; 54(8):1509-1513.

32. Cahn JW, Hagel W. Theory of the Pearlite Reaction. In: Zackay ZD, Aaaronson HI, editors. Decomposition of austenite by Diffusional Processes. New York: Interscience; 1960.

33. Vandermeer RA, Jensen DJ. Effects of nuclei clustering on recrystallization kinetics. Materials Science Forum. 2004; 467-470:193-196.

34. Meijering JL. Interface area, edge length, and number of vertices in crystal aggregates with random nucleation. Philips Research Reports. 1953; 8(4):270-290.

35. Gokhale AM. Calculation of Product Phase Grain Boundary Area During Solid State Transformations. Metallurgical Transactions A - Physical Metallurgy and Materials Science. 1988; 19A(9):2123-2131.

36. Gokhale AM. Calculation of Product Phase Grain Edge Length and Quadruple Points Per Unit Volume During Solid State Transformations. Metallurgical Transactions A - Physical Metallurgy and Materials Science. $1989 ; 20 \mathrm{~A}(3): 349-355$

37. Tong WS, Rickman JM and Barmak K. Evolution of perimeter fraction during a phase transformation. Acta Materialia. 2000; 48(5):11811186. 